\title{
BROAD-SPECTRUM CLINICAL APPLICATIONS OF A LOW-FIDELITY SIMULATOR: EMPHASIS ON FUNCTIONAL IMPAIRMENT
}

\author{
Henry J. Moller \\ Sleep Research and Human Performance Laboratory, \\ Department of Psychiatry \\ University Health Network \\ University of Toronto \\ Toronto, Canada \\ E-mail: henry.moller@uhn.on.ca
}

\begin{abstract}
Summary: The recently published $7^{\text {th }}$ edition of the Canadian Medical Association's guide "Determining Medical Fitness to Operate Motor Vehicles" places an increased emphasis on functional assessment rather than exclusively on a diagnostic categorization in determining license eligibility of Canadian drivers. While there is currently significant interest in research and development of simulation scenarios suitable to screen for specific medical impairments, our research group at University of Toronto has expanded the utility of a single lowcost low-fidelity interactive driving simulation paradigm to screen for functional impairments related to fitness-to-drive in a wide number of clinical conditions. The long-term objective of this project is to be able to use this simulation protocol beyond a research model in fitness-to-drive assessments performed in a routine clinical context.
\end{abstract}

\section{METHODS}

A normative database of driving performance in healthy control volunteers was developed. Thirty one healthy subjects with no significant sleep, medical and psychiatric pathology were assessed in a monotonous driving simulation paradigm requiring corrective steering maneuvers to simulated wind gusts randomized in direction and interval. A repeat-testing protocol of four separate 30-minute driving sessions at two-hourly intervals (10:00, 12:00, 14;00 and 16:00) was used, modeled after daytime polysomnography tests such as the Multiple Sleep Latency Test (MSLT) or Maintenance of Wakefulness Test (MWT). Convergent data sources included drivers' subjective ratings of sleepiness and alertness, EEG-verified microsleep episodes (MS), and a variety of real-time driving simulator performance measures such as speed, lane tracking, reaction time (RT) and off-road events (crashes). Performance of subsequent clinical cohorts were compared using parametric tests for continuous variables such as speed and RT, and nonparametric for discrete variables (i.e. crashes and MS).

\section{RESULTS}

The following mean performance variables were obtained for the normative sample: mean speed $=89.2 \mathrm{~km} / \mathrm{h}+/-4.5 \mathrm{~km} / \mathrm{h}$, mean speed variability: $0.7+/-4.3 \mathrm{~km} / \mathrm{h}$, lane tracking $28.9 \%+/-$ $4.1 \%$ (with $25 \%$ representing centre of right lane), reaction time $1.04+/-0.39 \mathrm{~s}$, mean crashes/drive $1.3+/-1.2$, mean microsleeps/drive $0.8+/-1.2$. Mean values for both discrete variables were noted to display significant skew towards "zero." A modest trend of increasing 
speeds with repeat drives was noted $(\mathrm{p}=.05$ in comparing 16:00 to 10:00 and 14:00 drives, N.S for other time comparisons), and a significant trend towards fastest RT during the first drive of the day ( $\mathrm{p}<.01$ for 10:00 drives compared to all other drives). Finally, crashes occurred significantly more frequently for the 16:00 compared to 10:00 drives $(p=.05)$, but did not reach significance for other time comparisons.

This normative cohort was organically intended by our laboratory to study driving performance of hospital patients with excessive daytime sleepiness (EDS). In a cohort of 27 "high-risk" patients with reported EDS (Epworth Sleepiness Scale score $>10$ and/or self-report of falling asleep while driving), specifically differences in RT, crash rate, and MS incidence were noted, with no significant differences for other variables including lane tracking. This was thought to be chiefly due to the nature of the embedded secondary performance task of required steering reaction to wind gusts, with intrusion of MS events mitigating impairment. Based on results of this study, "cut-off" values for impairment were established for simulator performance of mean $\mathrm{RT}>1.5$ and mean crash rate $>3$ per 30 minute simulation.

In a recent cross-sectional study comparing a group of 19 unmediated depressed patients to our normative cohort, we also found significantly increased RT and crash risk ( $\mathrm{p}<.05$ for both), although there was no significant circadian variation in performance noted. Presumably in this clinical cohort, relative task disengagement was due to psychomotor disturbance, motivational factors or preoccupation with depressive cognitions, and less due to sleepiness or MS intrusion, although it could be argued that sleep disturbance is also commonly associated with depression.

Stroke patients are now being studied in a clinical case-series format, with initial results suggesting that insight into impairment, as well as brain lesion location, plays a significant role in mitigating driving simulator performance, varying from severely impaired to comparable to normals. Pilot work in patients with mild dementia is now also commencing. Whereas it is a common clinical request by patients with organic brain disease (such as stroke) to have their driving privileges reinstated, participation in a standardized simulator protocol using values from normative patients has revealed a broad spectrum of performance and impairment.

\section{CONCLUSIONS}

Through generation of a normative cohort, it has been possible to study a number of clinical populations at potential risk of driving impairment. While neurocognitive performance deficits on a simple driving task were originally thought to be due to occurrence of MS episodes, a follow-up study in patients with depression but not EDS suggests that these lapses are not invariably associated with microsleeps, but may signify more functional disturbances of humanmachine interaction. The terms "absence" and "alertness" have been used by our research group to conceptualize the underlying factors that could play a role in reducing task immersion and resulting in episodic task disengagement, based on the presence-absence spectrum described in the Human Factors literature.

While actual intrusion of sleep-related EEG activity is probably easier to detect than subtle shifts in neural networks due to shifts in affective, attentional or motivational state, appreciating the fluctuating and non-continuous nature of the states of absence and presence can form the basis of 
a better understanding of variations in performance on simulated driving tasks. Furthermore, the issue of insight into impairment is relevant. If an individual subjectively feels that they are performing well on a task, yet there is a mismatch in terms of simulator performance, it might be advisable to consider the role of factors such as fatigue and sleepiness, particularly on prolonged task exposure. Use of subjective self-rating scales for perceived alertness, sleepiness, mood, attention and perceived global capacity for safe driving are useful routine adjuncts to simulator performance, and can often clarify the issue of insight into impairment in both organic and functional impairments.

In general, grossly impaired driving simulator performance is a useful "red flag" for further ergonomic/on-road evaluation (in the case of patients whose fitness-to-drive is in question but who have a license at the time of testing), or can increase the confidence of a clinician who is reluctant to give in to pressure by a patient to have a license reinstated. However, normal simulator performance does not necessarily translate to normal "real-world" performance, where difficult-to-control variables such as traffic volume, interaction with other drivers and distractions cause fluctuating cognitive and task-specific demands.

This broad-spectrum model of measuring driving impairment using simulators highlights current emphasis on the relevance of functional abilities versus diagnostic categorization, and has formed the basis of pilot work using our low-fidelity simulator model to study patients with driving impairments caused by both organic and functional neurocognitive impairments.

\section{ACKNOWLEDGMENT}

This research was in part funded by a postdoctoral research fellowship from the Canadian Institute of Health Research.

\section{REFERENCES}

Determining Medical Fitness to Operate Motor Vehicles: CMA Driver's Guide ( $7^{\text {th }}$ edition). Canadian Medical Association, 2006.

Moller, H.J., Kayumov, L., Bulmash, E.L., Nhan, J., Shapiro, C.M. (2006). Simulator performance, microsleep episodes and subjective sleepiness: normative data using convergent methodologies to assess driver drowsiness. Journal of Psychosomatic Research, 61(3):335- 342.

Bulmash, E., Moller, H.J., Kayumov, L., Wang, X., Shapiro, C.M. (2006). Psychomotor disturbance in depression: assessment using a driving simulator paradigm. Journal of Affective Disorders, 93, 213-8.

Chung, F., Kayumov, L., Sinclair, D., Edward, R., Moller, H.J., Shapiro, C.M. (2005). What is the Driving Performance of Ambulatory Surgical Patients after General Anesthesia? Anaesthesiology, 103(5), 951-6.

Nhan, J., Moller, H.J. (2006). Task fatigue and Driving Performance: How important is length of simulation? Cyberpsychology and Behavior (6), 705. 
Moller, H.J. (2006). The neurocognitive role of 'absence' in multimodal simulator protocols. Proceedings of the IEEE 2006 International Conference of the Engineering in Medicine and Biology Society, $5^{\text {th }}$ International Workshop on Virtual Rehabilitation, (G. Burdea, Ed). New York, 116- 120. 